EPJ manuscript No.

(will be inserted by the editor)

\title{
Interaction versus dimerization in one-dimensional Fermi systems
}

\author{
Cosima Schuster and Ulrich Eckern \\ Institut für Physik, Universität Augsburg, D-86135 Augsburg, Germany \\ the date of receipt and acceptance should be inserted later \\ Dedicated to J. Zittartz on the occasion of his 60th birthday
}

\begin{abstract}
In order to study the effect of interaction and lattice distortion on quantum coherence in one-dimensional Fermi systems, we calculate the ground state energy and the phase sensitivity of a ring of interacting spinless fermions on a dimerized lattice. Our numerical DMRG studies, in which we keep up to 1000 states for systems of about 100 sites, are supplemented by analytical considerations using bosonization techniques. We find a delocalized phase for an attractive interaction, which differs from that obtained for random lattice distortions. The extension of this delocalized phase depends strongly on the dimerization induced modification of the interaction. Taking into account the harmonic lattice energy, we find a dimerized ground state for a repulsive interaction only. The dimerization is suppressed at half filling, when the correlation gap becomes large.
\end{abstract}

PACS. 71.10.-w Theories and models of many electron systems - 75.10.Jm Quantized spin models

\section{Introduction}

Recent experiments on $\mathrm{CuGeO}_{3}$ [1] and $\mathrm{NaV}_{2} \mathrm{O}_{5}$ [2, 3], which show at low temperature a transition to a nonmagnetic ground state accompanied by a structural transition, led to renewed interest in spin-Peierls systems, as well as in the more general question of structure versus correlation induced metal-insulator transitions. The model of spinless fermions, which we consider here in detail, describes certain aspects of both, and it contains, as a special point in parameter space, the experimentally relevant isotropic Heisenberg antiferromagnet. In addition, the model contains, in the limit of an Ising type interaction, a szenario which is closer to that found for a more realistic model, including the electron spin, namely the Hubbard-Peierls model. Here we concentrate on the spinless fermion model, which nevertheless shows a surprisingly rich phase diagram (at zero temperature) as a function of interaction and dimerization.

In the next section, we introduce the various representations of the model. In Sect. 3, we quantitatively determine the extension of the delocalized phase, which was predicted for an intermediate attractive interaction. Thereby we identify the region where the fermion-phonon coupling is irrelevant, i.e. the ground state remains extended. In Sect. 4, we consider a repulsive interaction, where we find that a stable dimerization develops. For a very strong interaction the dimerization is reduced again, because conflicting ordering occurs. In the summary, Sect. 5, we present the $u$ - $V$ phase diagram, as well as the results for the dimerized state.

\section{The model}

As a starting point for the study of a general spin-Peierls system in one dimension, we consider an XXZ model with a dimerized interaction in a local magnetic field; the latter can be considered as a staggered magnetic field resulting from the surrounding chains [4], or resulting from random magnetic impurities. In the following, we concentrate on the dimerization-induced modification of the interaction, and, for comparison, include the magnetic field only occasionally. Furthermore, we neglect frustration effects which arise from next-nearest-neighbor couplings. Thus we start with the following Hamiltonian:

$$
\begin{aligned}
H_{\text {spin }}= & -\sum_{n=1}^{M} J_{n}(u)\left(\sigma_{n}^{x} \sigma_{n+1}^{x}+\sigma_{n}^{y} \sigma_{n+1}^{y}\right. \\
& \left.+\Delta \sigma_{n}^{z} \sigma_{n+1}^{z}\right)+\sum_{n=1}^{M}\left(-h_{n} \sigma_{n}^{z}+\frac{K_{0}}{2} u_{n}^{2}\right),
\end{aligned}
$$

where $J_{n}(u)=J\left(1-(-)^{n} \lambda_{J} u\right)$; the local lattice distortion, $u_{n}$, is assumed to be given by $u_{n}=2\left(x_{n}-x_{n}^{0}\right) / a=$ $(-1)^{n} u$. For the "clean" XXZ model, i.e. for $u=0, h_{n}=0$, one finds three phases: a ferromagnetic phase for $\Delta \geq 1$, a gapless phase for $-1 \leq \Delta<1$, whose low lying excitations are given by those of a Luttinger liquid, and an antiferromagnetic phase for $\Delta<-1$. The spin model is experimentally most relevant for $\Delta=-1$, i.e. the isotropic antiferromagnetic Heisenberg case. The corresponding fermion 
model is obtained via the Jordan Wigner transformation:

$$
\begin{aligned}
& \sigma_{m}^{-}=e^{-\mathrm{i} \pi \sum_{l=1}^{m-1} n_{l}} c_{m}, \quad \sigma_{m}^{+}=\left(\sigma_{m}^{-}\right)^{+}, \\
& \sigma_{m}^{z}=2 n_{m}-1, \quad n_{m}=c_{m}^{+} c_{m} .
\end{aligned}
$$

It is customary to change the notation: $J=t, J \Delta=$ $-V / 2$, and $\epsilon_{m}=-2 h_{m} ;$ furthermore we now assume that the coupling to (static) phonons can be varied independently in the hopping and the interaction. The result is the following:

$$
\begin{aligned}
H_{\text {fermion }}= & -\sum_{m} t_{m}(u)\left(c_{m}^{+} c_{m+1}+c_{m+1}^{+} c_{m}\right) \\
& +\sum_{m} V_{m}(u) n_{m} n_{m+1} \\
& +\sum_{m} \epsilon_{m} n_{m}+M \frac{K_{0}}{2} u^{2}
\end{aligned}
$$

where $t_{m}(u)=t\left(1-(-)^{m} \lambda_{t} u\right), V_{m}(u)=V\left(1-(-)^{m} \lambda_{V} u\right)$. Furthermore, we consider twisted boundary conditions, $c_{0}=\mathrm{e}^{\mathrm{i} \phi} c_{M}$. The length of the chain is $L=M a$, the number of electrons $N$, and the filling $n_{0}=N / M$. In addition, we restrict ourselves to half filling, and set $t=1$ and $\lambda_{t}=1$ in some of the formulas below. The corresponding instabilities in the fermion model are at $V=-2$, towards a phase separated state, and at $V=2$, to a charge density wave (CDW) state. (Note that at $V=2$, the $4 k_{F^{-}}$ backscattering process becomes relevant [0].) Numerically this CDW state is difficult to determine, because, for finite systems, the hopping lifts the twofold degeneracy of the ground state via a symmetric superposition, and a uniform density is found. For the analytic considerations, we use the fact that the system is a Luttinger liquid in the gapless phase, and that the dimerization can be considered a perturbation. In the bosonized form [6], the Hamiltonian can be written as follows:

$$
\begin{aligned}
H_{\text {boson }} & =\int \frac{\mathrm{d} x}{2 \pi}\left\{\frac{v}{g}\left[\partial_{x} \varphi(x, t)\right]^{2}+\frac{1}{v g}\left[\partial_{t} \varphi(x, t)\right]^{2}\right. \\
& \left.+\frac{2 \pi \lambda u}{l_{0}} \sin [2 \varphi(x, t)]-\frac{\pi V a}{l_{0}^{2}} \cos [4 \varphi(x, t)]\right\},
\end{aligned}
$$

where $\lambda=\lambda_{t} t+\lambda_{V} V n_{0} / 2$, and $l_{0}$ is a short distance cut-off, $\approx 2 a$. Only the first order correction to the $2 k_{F^{-}}$ process, $\propto V u$, has been included. The Fermi velocity is given by $v=[\pi t \sin (2 \eta)] /(\pi-2 \eta)$, and $g=\pi / 4 \eta$, where $\eta$ parameterizes the interaction according to $V=-2 t \cos (2 \eta)$. Furthermore, the density is given by

$$
\frac{1}{2} \sigma_{n=x / a}^{z}=\rho(x)-n_{0}=\frac{\partial_{x} \varphi}{\pi}+\frac{2}{l_{0}} \cos \left(2 k_{F} x+2 \varphi\right) .
$$

Clearly, the term $\propto \cos [4 \varphi(x, t)]$, describing $4 k_{F}$ scattering processes, is responsible for the CDW ordering. For completeness we like to state that some properties of the dimerized Heisenberg chain, especially the spectrum, can also be obtained 77 from the equivalent relativistic model, the massive Thirring model.

The numerical results, presented below, are obtained on the basis of the XXZ model, Eq. (17).

\section{Phase transition at attractive interaction}

The first conjecture that the dimerized XXZ model undergoes a transition from the dimerized spin singlet phase to a phase with free spins at $\Delta=\sqrt{2} / 2$, was given in [8] based on a mapping onto the Ashkin-Teller-model. There, an estimate of the phase boundary, based on a series analysis, has been given. (Note the different sign convention.) For the XXZ model in a staggered magnetic field, Alcaraz [9] again found this critical point on the basis of predictions of conformal field theory. Thus it seems that the periodic distortion of the $\sigma^{z} \sigma^{z}$-interaction does not change the point of the transition. In the case of the bosonic model, assuming an interaction of the form $\sum U_{n} \cos n \varphi$, one finds the following renormalization group equations [10]:

$$
\frac{\mathrm{d} U_{n}}{\mathrm{~d} l}=U_{n}\left(2-\frac{n^{2}}{4} g\right)+\frac{1}{2} \sum_{n_{1}+n_{2}=n} U_{n_{1}} U_{n_{2}} .
$$

The critical value or a model containing only a single periodic term, $g=8 / n^{2}$, is in accordance with the exact solution of the sine-Gordon model [11,12]. One concludes that for the "clean" case $(u=0)$, there exists an instability for $g=1 / 2$, i.e. $V=2$. Consequently, considering the $\sin (2 \varphi)$-contribution in (何), we expect interesting effects for $g=2$, i.e. $V=-\sqrt{2}$, provided the dimerization, $u$, assumes a finite value.

Applied to our model, the critical behavior near the transition between a localized and a delocalized state is determined by the Berezinskii-Kosterlitz-Thouless equations [13]:

$$
\begin{gathered}
\frac{\mathrm{d} u}{\mathrm{~d} l}=u(2-g) \\
\frac{\mathrm{d} g}{\mathrm{~d} l}=-\frac{1}{2} g^{2} \lambda^{2} u^{2} .
\end{gathered}
$$

Considering the bosonized Hamiltonian, the equivalence of the "staggered" and "dimerized" XXZ model is also apparent: For the dimerized model, the relevant operator is proportional to $u \sin 2 \varphi$, while for a staggered field, it is $\propto h \cos 2 \varphi$. The phase shift of $\pi / 2$ corresponds to the coupling to the bond (in the hopping term) versus coupling to the site. As a consequence, the ground state for the dimerized model is a spin singlet, with $\varphi_{\min }=\pi / 2$ and $\sigma_{n}^{z}=0$, while in a staggered magnetic field, an antiferromagnetic ground state, with $\varphi_{\min }=0$ and $\sigma_{n}^{z}=(-1)^{n}$, is obtained. The critical behavior, however, is the same for both models.

We numerically verify these predictions and also determine quantitatively the phase boundary between the localized and the delocalized phase. In addition to the critical point at $u=0, g=2$, physical arguments suggest that the delocalized phase should not exceed the point $u=1, V=-2$ : the transition at $V=-2$, being first order, is stable against perturbations [8], and for $\lambda_{t} u=1$ every second bond is cut and therefore the system clearly is localized. Compared with the random disorder case [14], one is tempted to expect - incorrectly - that the delocalized phase is smaller. For the random case, this is due to a 
stronger renormalization of $g$, namely $\mathrm{d} g / \mathrm{d} l=-0.5 g^{2} W$ [15]; compare this with equation (8).

We will use the phase sensitivity, i.e. the reaction of the system to a change in the boundary condition, to determine this transition numerically for systems with finite size. The ground state energy $E(\phi)$ depends on the boundary condition, expressed via the phase $\phi$. In particular, we determine below the energy difference between periodic and anti-periodic boundary conditions, $\Delta E=$ $(-)^{N}(E(0)-E(\pi))$. Recall that, for a clean system, the ground state energy and the finite size corrections can be obtained from the Bethe Ansatz [16]. At half filling (and for odd particle number), the result, in the Luttinger regime, is given by 17

$$
E_{M}(\phi)-M \varepsilon_{\infty}=-\frac{\pi v}{6 M}\left(1-3 g \frac{\phi^{2}}{\pi^{2}}\right),
$$

where $\varepsilon_{\infty}$ is the energy density in the thermodynamic limit. Thus $M \Delta E=\pi v g / 2$, independent of $M$, for the metallic state. In an insulator, on the other hand, the system cannot react to a twist in the boundary condition, i.e. $M \Delta E$ is expected to decrease with system size.

For non-interacting fermions, the finite size corrections for the Su-Schrieffer-Heger (SSH) model 18], $\varepsilon_{\infty}(u) \propto$ $u^{2} \ln u$, can be determined in an elementary way, with the help of the Euler-McLaurin formula. However, it is necessary to handle the van Hove singularity at the band edge carefully for periodic boundary conditions, while for antiperiodic boundary conditions, this term is absent. With $h=2 \pi / M$, we obtain the following result:

$$
\begin{aligned}
E_{M}(u)-M \varepsilon_{\infty}(u) & =\left(\frac{u^{2}}{2 h} \ln u-\frac{u^{2}}{2 h} \ln \left(\sqrt{u^{2}+h^{2}}-h\right)\right. \\
& \left.-\frac{1}{2} u+\frac{1}{12} \frac{h^{2}\left(1-u^{2}\right)}{\sqrt{h^{2}+u^{2}}}-\frac{1}{12} h\right) .
\end{aligned}
$$

Thus, for small systems, $h \gg u$, we obtain a correction linear in $u$ which does not depend on the system size, and a correction to the $u^{2} \ln u$-term:

$$
\approx-\frac{1}{2} u-\frac{u^{2}}{2 h} \ln \frac{u}{2 h}
$$

which we plot in figure 1. For large systems, $h \ll u$, the corrections are of order $1 / M$ :

$$
\approx-\frac{1}{12} h+\frac{1}{12} \frac{h^{2}}{u}
$$

Thus, for small enough systems, or small enough $u$, the phase sensitivity is $M \Delta E(u) \propto-u M$; this behavior results from the zone boundary contribution.

For $V \neq 0$, we combine the treatment of Loss 19 of a Luttinger liquid on a closed ring with the scaling equation (7), and a first order perturbative calculation, with the following result:

$$
M \Delta E(\lambda u)=M \Delta E(\lambda u=0)-\lambda u \frac{2 \pi}{v}\left(\frac{M}{M_{0}}\right)^{2-g} .
$$

This is similar to the case of a single impurity [14], but valid in a restricted regime only. This estimation cannot be extended to large $M$ or $u$, because, in a finite system with a gap $\Delta(u)$, the "free motion" is only seen if $L / \xi \propto M \Delta(u) \propto M u$ is small. For larger systems, the phase sensitivity depends exponentially on the correlation length, as discussed in [20]. In addition, we have neglected the renormalization of the interaction parameter $g$, see equation (8), which is reasonable only for $V<0$, as can be seen easily by integrating the RG equations (7) and (8). For example, only the samples with $u=0.01$, for system sizes $M=24$ and $M=50$, can be fitted with equation (11). Already for $u=0.05$ and $M=24$ deviations from the first order treatment are obvious; compare figure 2 .

Our aim is to determine quantitatively the phase boundary for the dimerized XXZ model $\left(\lambda_{V}=\lambda_{t}\right)$, and to compare the results with those in the case of $\lambda_{V}=0$. In the latter case, one may compare with results obtained from the massive Thirring model, or the RG equations. In our density matrix renormalization group calculations [21], we kept 1000 states and performed 9 finite lattice sweeps. First, consider $\lambda_{V}=0$. In order to determine the phase transition near $u=0$, we plot in figure 3 the phase sensitivity for $M=50$ and 100 , and small $u$, and confirm that the transition occurs near $V \approx-1.4$. (The transition point is defined as the point where the phase sensitivity becomes independent of the system size.) Proceeding to higher values of $u$, we can follow the phase boundary, as shown in figure A. Already at $u=0.4$, we find a completely localized phase, and we verify the presumption that the delocalized phase extends to the line $V=-2$. In figures 5 and 6, we show the difference between the two cases, $\lambda_{V}=\lambda_{t}$ versus $\lambda_{V}=0$. There is no drastic change in the qualitative behavior for small $u$, and the transition point remains, as expected from (4) and the renormalization group equation (7), at $V \approx-\sqrt{2}$. We also find, in the case $\lambda_{V}=\lambda_{t}$, that the phase sensitivity decreases more slowly for an attractive interaction, and faster for a repulsive interaction. Increasing the dimerization further, we see that the delocalized phase is considerably larger for $\lambda_{V}=\lambda_{t}$, and indeed extends to the point $u=1$. In comparison with the disordered model [14, the transition occurs at a stronger interaction, and the delocalized region extends to the line $V=-2$.

\section{Interaction and dimerization}

In the last section, we determined the $u$ - $V$ phase diagram, under the assumption that the lattice distortion is a fixed parameter. In a more realistic model, the harmonic lattice energy will compensate the energy gain of the fermions or the spins, respectively, resulting in a finite equilibrium value of $u$.

We consider the ground state energy for periodic boundary conditions, studying the lattice effect for $-2<V<2$ first. The numerical data (see figures 7 and 8), obtained for 100- or 200-site systems, while keeping about 300 to 400 states (for about 100 sites the finite size corrections 
are very small and the term linear in $u$ is absent), show that we can distinguish three regimes. For $V$ between -2 and 0 , the gain in the fermionic energy grows parabolically, $\Delta E(\lambda u) \propto-(\lambda u)^{2}$. For $0<V<2$, the energy gain is stronger, $\propto u^{\alpha}$, with an exponent which reaches its minimum of $4 / 3$ at $V=2$, where correlations change the behavior.

Explicitly, from an analysis of the massive Thirring model, one finds 222 the energy gain, $\Delta E(\lambda u)=E(\lambda u)-$ $E(u=0)$, to be given by

$$
\begin{aligned}
-\sqrt{2}<V<0: & \Delta E(\lambda u)=c_{1} u^{\alpha}-c_{2} u^{2}, \\
& \alpha=2 /(2-g)>2 \\
0<V \leq 2: & \Delta E(\lambda u)=-c_{1} u^{\alpha}+c_{2} u^{2} \\
& 4 / 3<\alpha<2
\end{aligned}
$$

with $c_{1}>c_{2}>0$; compare figure 9 . The non-quadratic term can also be obtained from a self-consistent treatment of the perturbation. In this so-called self-consistent harmonic approximation (SCHA) 11,23 an optimal quadratic approximation for the periodic potential around one of its minima is chosen, which corresponds to the following replacement:

$$
U \cos n \varphi \rightarrow U\left[1-\frac{n^{2}}{2}\left(\varphi^{2}-\left\langle\varphi^{2}\right\rangle\right)\right] e^{-n^{2}\left\langle\varphi^{2}\right\rangle / 2}
$$

here, explicitly,

$$
\left\langle\varphi^{2}\right\rangle=\frac{g}{2} \ln \frac{2 \pi}{l_{0} q_{0}}, \quad q_{0}^{2}=\frac{n g U}{v} e^{-n^{2}\left\langle\varphi^{2}\right\rangle / 2}=\frac{\Delta^{2}}{v^{2}} .
$$

The quadratic term in equation (12) is related to the tunneling between the minima of the periodic potential [24]. The logarithmic dependence on $u$ for $V=0$, which was found in 18, is contained in the above formulas:

$$
\begin{aligned}
& V=0: \Delta E_{0}=-\frac{M t}{\pi}(\lambda u)^{2}\left(\ln \frac{4}{\lambda u}-0.5\right) \\
& V \approx 0: \Delta E=\Delta E_{0}-\frac{M V}{\pi}(\lambda u)^{2} \ln ^{2} \frac{4}{\lambda u} .
\end{aligned}
$$

Adding the harmonic lattice energy, $M K_{0} u^{2} / 2$, we realize that a finite equilibrium dimerization cannot be be stabilized for an attractive interaction. For small $|V|$, the second logarithmic term in (15) always leads to a positive slope of $\Delta E(\lambda u)$ at $u=0$; and for large (negative) $|V|$, we find, as shown in the previous section, a delocalized phase for small $u$.

For the non-interacting case $(V=0)$, the stable dimerization $\lambda u_{0}$ and the energy gain are given by well-known expressions [23] for CDW-systems. With $\gamma=\lambda^{2} t / K_{0}$, the results are

$$
\begin{aligned}
\lambda u_{0} & \propto \mathrm{e}^{-1 / \gamma}, \\
\Delta\left(\lambda u_{0}\right) & \propto \mathrm{e}^{-1 / \gamma}, \\
E_{\text {total }}\left(\lambda u_{0}\right) & \propto \mathrm{e}^{-2 / \gamma} .
\end{aligned}
$$

For a repulsive interaction, we find the following algebraic dependences:

$$
\begin{aligned}
\lambda u_{0} & \propto \gamma^{\frac{2-g}{2-2 g}}, \\
\Delta\left(\lambda u_{0}\right) & \propto \gamma^{\frac{1}{2(1-g)}}, \\
E_{\text {total }}\left(\lambda u_{0}\right) & \propto \gamma^{\frac{1}{1-g}},
\end{aligned}
$$

and, for example,

$$
\begin{array}{lll}
V=1: & \lambda u_{0} \propto \gamma^{5 / 2}, & E_{\text {total }}\left(\lambda u_{0}\right) \propto \gamma^{4} \\
V=2: & \lambda u_{0} \propto \gamma^{3 / 2}, & E_{\text {total }}\left(\lambda u_{0}\right) \propto \gamma^{2} .
\end{array}
$$

Another interesting quantity is the curvature of the total energy at the minimum, which is related to the zone boundary phonon frequency, $\omega_{2 k_{F}}$. For a decoupled system $(\lambda=0)$, the curvature is given by $K_{0}$, the bare value. The fermion-lattice coupling changes this quantity as follows:

$$
\begin{array}{ll}
V=0: & E_{\text {total }}^{\prime \prime}\left(u_{0}\right) / M=\gamma K_{0} \\
V>0: & E_{\text {total }}^{\prime \prime}\left(u_{0}\right) / M=K_{0}(2-\alpha) .
\end{array}
$$

We thus conclude that for interacting fermions, the softening of the phonon at the zone boundary does not depend on the coupling strength $\gamma$, but on the exponent of the energy gain of the fermions only.

Next we consider the regime $V>2$, where a correlation gap develops, for the "clean" case given by [25]

$$
\begin{aligned}
V \gtrsim 2: & \Delta(V) \propto e^{-\pi^{2} / \sqrt{V-2}}, \\
V \rightarrow \infty: & \Delta(V) \propto V .
\end{aligned}
$$

A gap also develops in a frustrated model, i.e. when nextnearest-neighbor coupling is included, for $V=2$ and provided that $V_{\text {frust }}>V_{\text {frust }}^{c}$. This is a consequence of a relevant $4 k_{F}$-backscattering process, and believed to be relevant for $\mathrm{CuGeO}_{3}$ [26 27]. As shown in [28], a $\cos 4 \varphi$-term leads to a Neel (Ising-type) or a dimer (frustration) ground state, respectively, depending on its sign. Thus we expect that a next-nearest-neighbor interaction will increase the dimerization, while an Ising-type interaction reduces it. Possible distinctive features between the frustration induced and the externally, by $\lambda u$, driven dimerized state are discussed in [29].

Considering the numerical data for a strong repulsive interaction, $V>2$, see figure \&, the energy gain is nearly constant at first, when increasing $V$; only the prefactor is slightly reduced. Arguments supporting this observation are given below. Increasing the interaction, the energy gain is drastically reduced, the exponent approaches again 2 , and therefore no minimum is found in the total energy. To understand this behavior, we consider in more detail the extrema of the combined sin-cos-potential, $U(\varphi)=-\tilde{V} \cos 4 \varphi-\tilde{u} \sin 2 \varphi$, where $\tilde{u}=-2 \pi v g \lambda u / l_{0}$, and $\tilde{V}=\pi v g V / l_{0}^{2}$. For a large interaction, the minima are at $\sin 2 \varphi_{0}=\tilde{u} /(4 \tilde{V})$, and the expansion reads

$$
U(\varphi) \approx-\tilde{V}-\frac{\tilde{u}^{2}}{8 \tilde{V}}+\left(8 \tilde{V}-\frac{\tilde{u}^{2}}{2 \tilde{V}}\right)\left(\varphi-\varphi_{0}\right)^{2} .
$$


So for a strong interaction, it is possible to consider the $u^{2}$-terms in the potential as a small perturbation to the exact results [25]. Note that, for a strong interaction (i.e. the renormalized $g$ is $\approx 0$ ), fluctuations are not important since $\left\langle\varphi^{2}\right\rangle \approx 0$. These arguments support the result, obtained numerically, that the energy gain is quadratic in $u$. For large $u$, on the other hand, the minima are located at $\cos 2 \varphi_{0}=0$, and the expansion for small interaction reads

$$
U(\varphi) \approx(\tilde{V}-\tilde{u})+2(\tilde{u}-4 \tilde{V})\left(\varphi-\varphi_{0}\right)^{2}
$$

For an interaction $V \approx 2$, where the effective influence of the $\tilde{V} \cos 4 \varphi$-term is expected to be small, we repeat the perturbation analysis, i.e., we evaluate first the $\tilde{u} \sin 2 \varphi$ term within the SCHA; thus $\Delta(u) \propto u^{2 / 3}$. Adding then the interaction term to the gapped system, and taking into account the fluctuations, the dimerization gap is reduced:

$$
\Delta^{2}(u) \rightarrow \frac{\Delta^{2}(u)}{(1+\bar{V})}, \quad \bar{V}=\frac{V}{\pi v} .
$$

For a frustrating interaction, there is no change in the minima, and we find, for a small frustration, a similar expression to (22), and for a stronger frustration, a linear increase in $u$.

To check the validity of the perturbation analysis at $V \rightarrow 2$, we compare with results from the SCHA and from field theory. In the evaluation of the SCHA scheme, which considers both perturbations on an equal footing following (21), we find the following gap equation, which can be solved for $g=1 / 2$ :

$$
\Delta^{2}=\tilde{u}\left(\frac{l_{0} \Delta}{2 \pi v}\right)^{g}-4 \tilde{V}\left(\frac{l_{0} \Delta}{2 \pi v}\right)^{4 g}
$$

the result is:

$$
\Delta(u, \bar{V})=\frac{\Delta(u)}{(1+\bar{V})^{2 / 3}} .
$$

Clearly, the exponent of the denominator does not coincide with the one in (22).

However, a more careful treatment, particularly with respect to the marginality of $\cos 4 \varphi$ at $V=2$, introduces a logarithmic correction to the dimerization gap [4], given by

$$
\Delta(u, \bar{V}) \propto \frac{\Delta(u)}{[1+\bar{V} \ln (v / \Delta(u))]^{1 / 2}} .
$$

This logarithmic term is a consequence of the scaling of the marginal operator $V(L)=V(1+\pi V \ln L)^{-1}$, see [30], and therefore is not found within both of the above considered approximations. The remaining difference between (24) and (25), namely the different exponents, can be explained by higher order contributions 30]. Thus the perturbation approach, which led to (22), is rather close to the correct result, (25), and even the simple SCHA is not too far off, as it shows that the interaction essentially changes the prefactor, i.e. the gap is $\sim \Delta(u)$.
The observation that a repulsive interaction in the onedimensional Hubbard model can enhance the dimerization 31 can be explained now in the following way: For small $\bar{U}$, where the charge gap is small, we find an energy gain $E_{\text {charge }} \propto E_{\text {spin }} \propto t u^{4 /\left(2-g_{c}\right)}$, with the corresponding interaction parameters $g_{s}=1$ for the spin and $g_{c}$ for the charge. At half filling, an intermediate $U$ leads to a large enough gap to push down the dimerization in the charge channel, but in the spin channel, no gap appears, and the system gains energy from the coupling to the lattice. The charge gap reduces, nevertheless, the prefactor from $t$ to $t^{2} / U$.

\section{Summary}

In summary, we have shown that a periodic distortion of the lattice is irrelevant for $V<-\sqrt{2}$, resulting in a delocalized phase. The transition point, $V_{c}(u=0)=-\sqrt{2}$, is not modified by the way of coupling to the lattice. However, the extension of the delocalized phase depends strongly on the coupling to the interaction, i.e. the parameter $\lambda_{V}$. For the spin model $\left(\lambda_{V}=\lambda_{t}\right)$, the delocalized region is considerably larger than for the case $\lambda_{V}=0$. In comparison with a random disorder model [14], the most important difference is the fact that the delocalized region extends down to the line $V=-2$. The phase diagram is summarized in figure 10. Due to the difficulties in determining precisely the point where the curves for different system sizes coincide, we can plot only a rough phase diagramr; nevertheless the transition seems to be steeper for $\lambda_{V}=\lambda_{t}$, compared to the case $\lambda_{V}=0$.

Including the harmonic lattice energy, we find that the dimerized state is stable, i.e. the total energy develops a minimum at a finite $u_{0}$, for a repulsive interaction only. With increasing interaction, the dimerization increases even further, provided no interaction induced, competing ordering occurs. A schematic plot is given in figure 11. Furthermore, already at $V=4$, no equilibrium dimerization develops, as can be seen from the preliminary data shown in figure 12, where we plot the gap versus inverse system size. (The lines are obtained from a polynomial fit in $1 / M$ for the case $u=0$, and an exponential fit as mentioned in [32, respectively.) Obviously, no additional gap from the dimerization is seen for $V=4$. In contrast, for smaller $V$, especially $V=2$, the dimerization contribution is significant.

We thank Peter Schmitteckert for providing us with the DMRG algorithm, and Peter Schwab for useful discussions. This work was supported by the Deutsche Forschungsgemeinschaft (Forschergruppe HO 955/2-1). The calculations were mostly done on the IBM SP2 at the Leibniz-Rechenzentrum in Munich.

\section{References}

1. M. Hase, I. Terasaki, and K. Uchinokura, Phys. Rev. Lett. 70, 3651 (1993). 
2. M. Isobe and Y. Ueda, J. Phys. Soc. Jap. 65, 1178 (1996).

3. M. Weiden, R. Hauptmann, C. Geibel, F. Steglich, M. Fischer, P. Lemmens, and G. Güntherodt, Z. Phys. B 103, 1 (1997).

4. H. J. Schulz, Phys. Rev. Lett. 77, 2790 (1996).

5. F. D. M. Haldane, Phys. Rev. Lett. 45, 1358 (1980).

6. F. D. M. Haldane, Phys. Rev. Lett. 47, 1840 (1981).

7. G. S. Uhrig and H. J. Schulz, Phys. Rev. B 54, R9624 (1996).

8. M. Kohmoto, M. den Nijs, and L. P. Kadanoff, Phys. Rev. B 24, 5229 (1981).

9. F. C. Alcaraz and A. L. Malvezzi, J. Phys. A: Math. Gen. 28, 1521 (1995).

10. Ines Safi, private communication.

11. R. F. Dashen, B. Hasslacher, and A. Neveu, Phys. Rev. D 11, 3424 (1975).

12. E. K. Sklyanin, L. A. Takhtajan, and L. D. Fadeev, Theor. Math. Phys. 40, 194 (1979).

13. J. Zang, A. R. Bishop, and D. Schmeltzer, Phys. Rev. B 52, 6723 (1995).

14. P. Schmitteckert, T. Schulze, C. Schuster, P. Schwab, and U. Eckern, Phys. Rev. Lett. 80, 560 (1998).

15. T. Giamarchi and H. J. Schulz, Phys. Rev. B 37, 325 (1988).

16. F. Woynarovich and H. P. Eckle, J. Phys. A 20, L97 (1987).

17. B. S. Shastry and B. Sutherland, Phys. Rev. Lett. 65, 243 (1990); B. Sutherland and B. S. Shastry, Phys. Rev. Lett. 65, 1833 (1990).

18. W. P. Su, J. P. Schrieffer, and A. J. Heeger, Phys. Rev. B 22, 2099 (1980).

19. D. Loss, Phys. Rev. Lett. 69, 343 (1992).

20. R. J. Baxter, Exactly Solved Models in Statistical Mechanics (Academic, New York, 1982).

21. S. R. White, Phys. Rev. Lett. 69, 2863 (1992); for the implementation see: P. Schmitteckert and U. Eckern, Phys. Rev. B 53, 15397 (BR) (1996).

22. T. Nakano and H. Fukuyama, J. Phys. Soc. Jap. 50, 2489 (1981).

23. H. Fukuyama and H. Takayama, in Electronic Properties of Inorganic Quasi-One-Dimensional Compounds, edited by P. Monceau (D. Reidel, Dordrecht, Holland, 1985), part I, p. 41.

24. F. Guinea, G. Gómez-Santos, M. Sassetti, and M. Ueda, Europhys. Lett. 30, 561 (1995); G. Gómez-Santos, Phys. Rev. Lett. 76, 4223 (1996).

25. R. Shankar, Int. J. Mod. Phys. B 4, 2371 (1990).

26. G. Castilla, S. Chakravarty, and V. J. Emery, Phys. Rev. Lett. 75, 1823 (1995).

27. J. Riera and A. Dobry, Phys. Rev. B 51, 16098 (1995).

28. F. D. M. Haldane, Phys. Rev. B 25, 4925 (1982).

29. R. Chitra and T. Giamarchi, Phys. Rev. B 55, 5816 (1997).

30. I. Affleck, D. Gepner, H. J. Schulz, and T. Ziman, J. Phys. A: Math. Gen. 22, 511 (1989).

31. V. Waas, H. Büttner, and J. Voit, Phys. Rev. B 41, 9366 (1990).

32. G. Bouzerar, A. P. Kampf, and G. I. Japaridze, condmat/9801046.

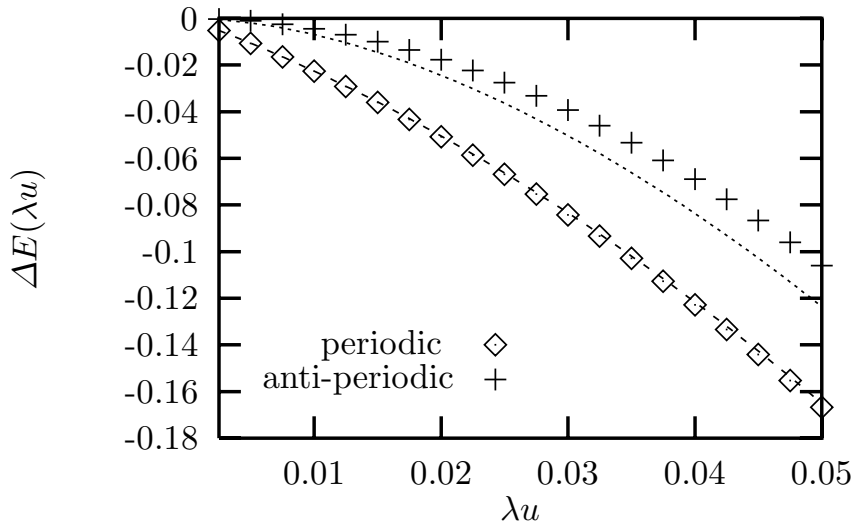

FIG. 1 . Finite size corrections to the ground state energy for $V=0$. The points $(\diamond,+)$ are numerical data for a 40 -site system, with periodic or anti-periodic boundary conditions. The dotted line shows the ground state energy in the thermodynamic limit, $\propto M u^{2} \ln u$, the dashed line is the energy with the corrections according to equation (10).

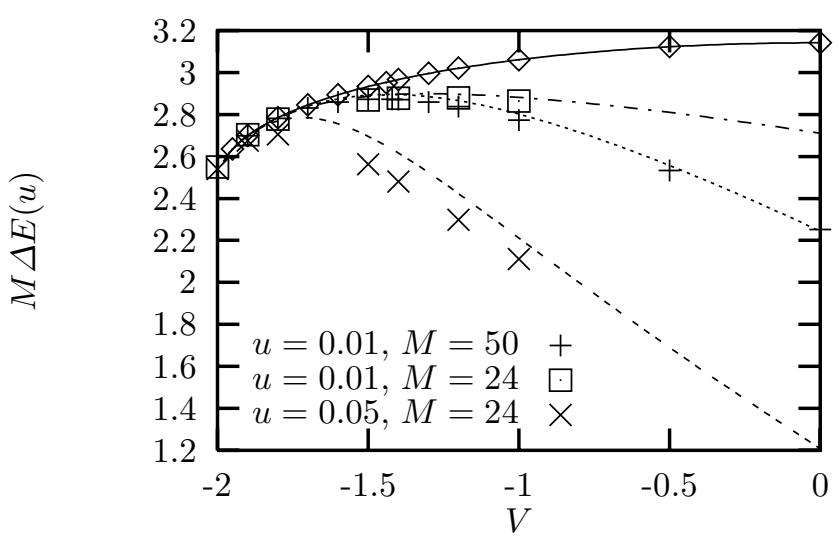

FIG.2: Comparison of numerical data with equation (11); $\lambda_{t}=1, \lambda_{V}=0, M_{0} \approx 2$. The $\diamond$ are numerical data for $u=0$ and $M=50$, in apparent agreement with the analytical result, equation (9) (full line).

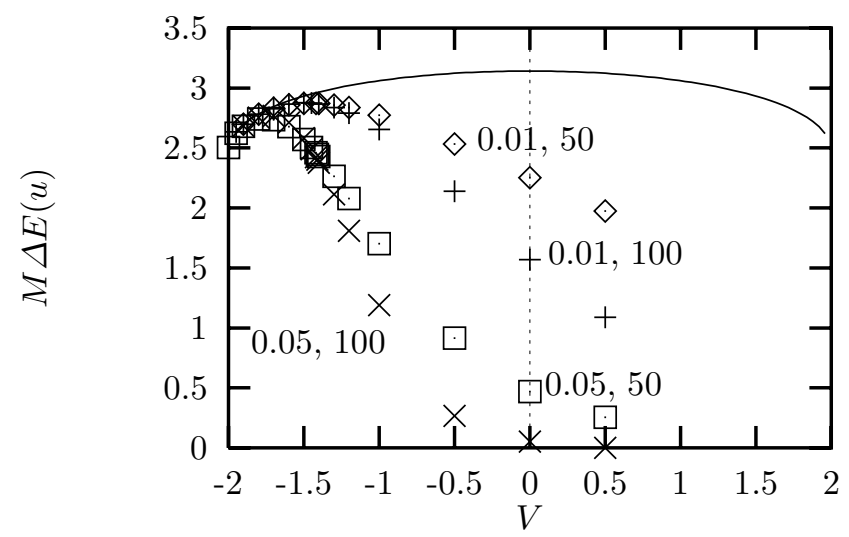

FIG. 3: Phase sensitivity versus interaction, for system sizes 50 and 100; $u=0.01$ and $0.05, \lambda_{t}=1, \lambda_{V}=0$. For comparison, we include here and in figures $4-6$, the clean limit result (full line). 


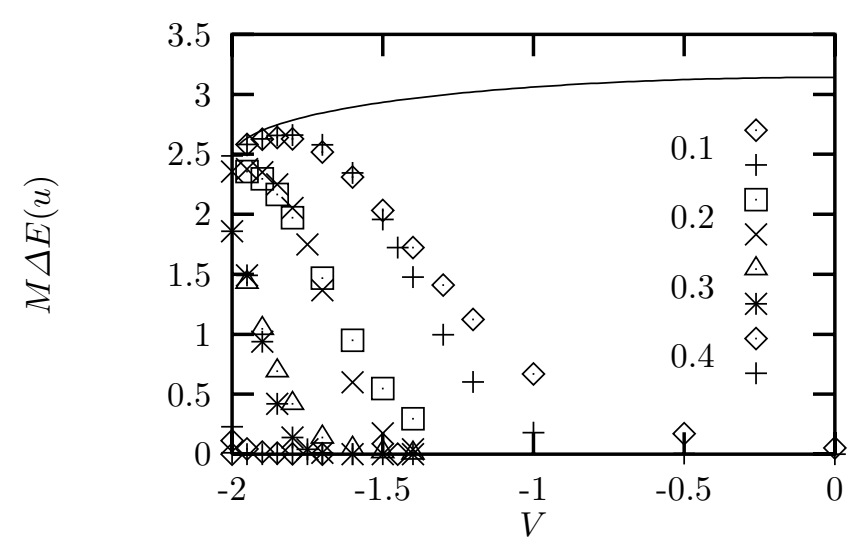

FIG. 14: Phase sensitivity versus interaction, for $u=$ $0.1 \ldots 0.4 ; \lambda_{t}=1, \lambda_{V}=0$. The respective upper data correspond to $M=50$, the lower ones to $M=100$.

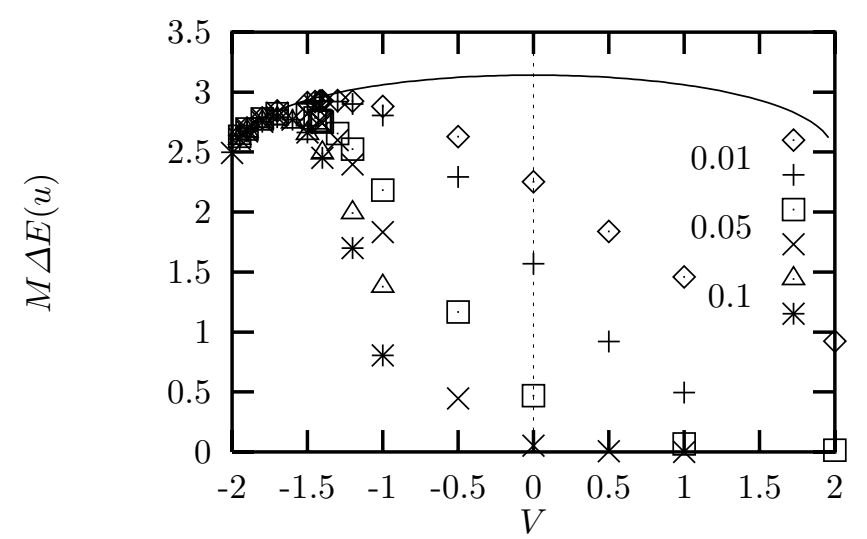

FIG. 5: Phase sensitivity versus interaction, for $u=$ $0.01 \ldots 0.1 ; \lambda_{V}=\lambda_{t}=1$. The respective upper data correspond to $M=50$, the lower ones to $M=100$.

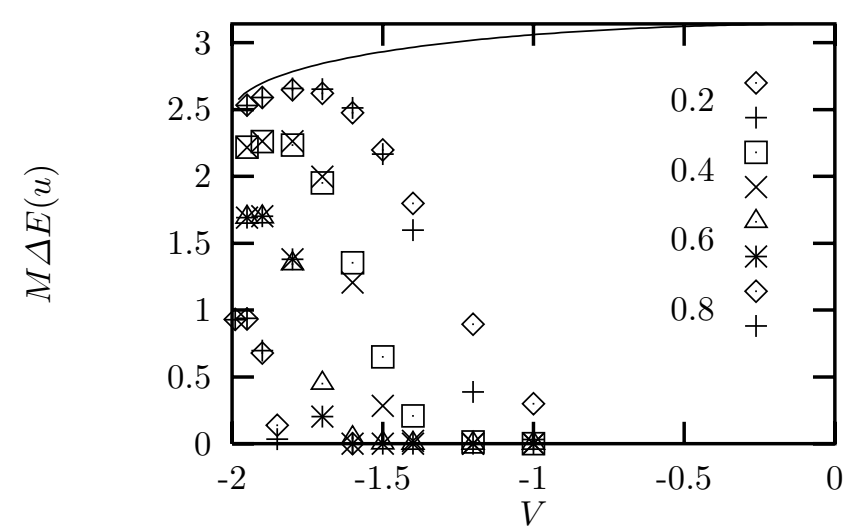

FIG. 6: Phase sensitivity versus interaction, for $u=$ $0.2 \ldots 0.8 ; \lambda_{V}=\lambda_{t}=1$. The respective upper data correspond to $M=50$, and the lower ones to $M=100$.

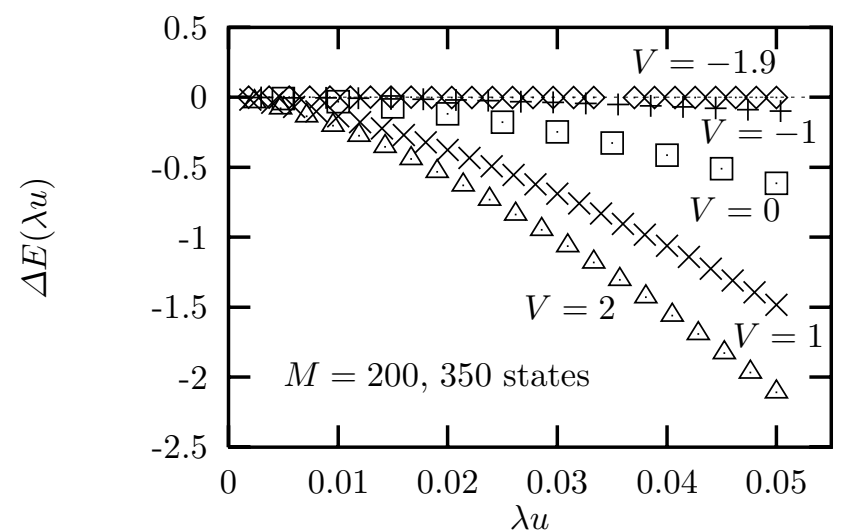

FIG. 7: Ground state energy versus $\lambda u$. The system size is $M=200$.

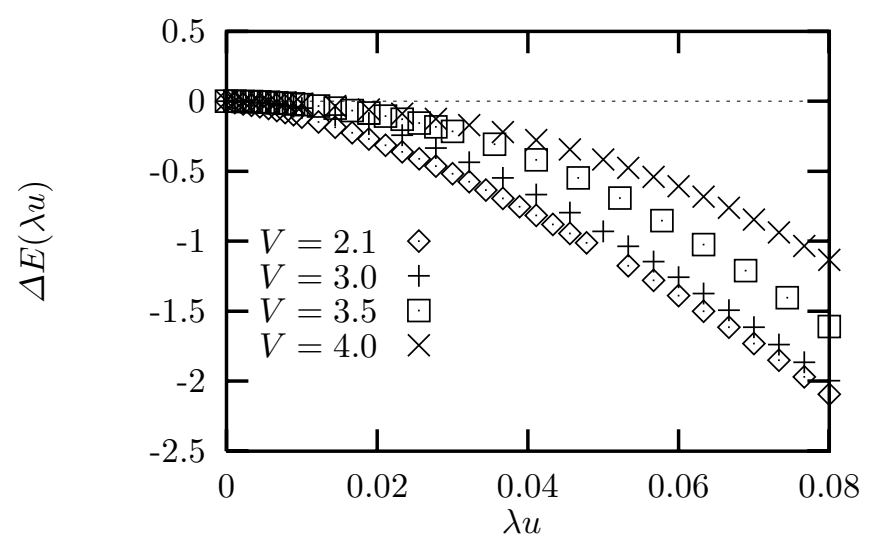

FIG. 8: Ground state energy versus $\lambda u$. The system size is $M=100$.

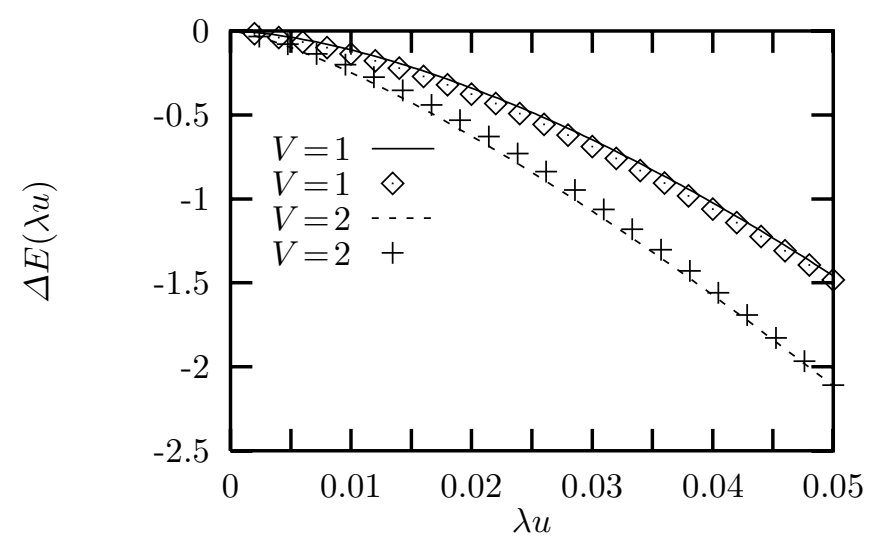

FIG. 9: Numerical versus analytic results. The full line corresponds to $\Delta E(\lambda u)=-E_{0}\left[1.3(\lambda u)^{8 / 5}-0.1(\lambda u)^{2}\right]$, the dashed line to $\Delta E(\lambda u)=-E_{0}\left[0.87(\lambda u)^{4 / 3}-0.044(\lambda u)^{2}\right]$, where $E_{0}=2 M t / \pi$. 


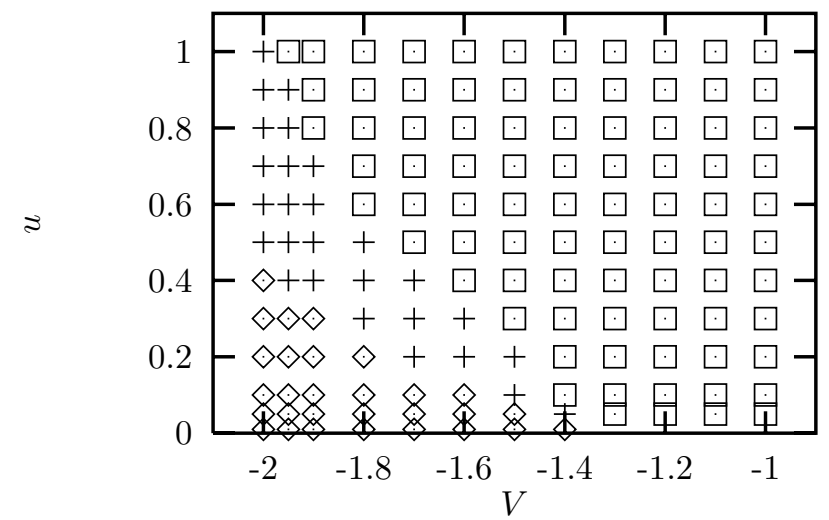

FIG. 10: Phase diagram. The symbol $\diamond$ denotes the delocalized region for $\lambda_{V}=0$. The delocalized region increases when $\lambda_{V}=\lambda_{t}$, as indicated by the + . The localized region is marked by $\square$.

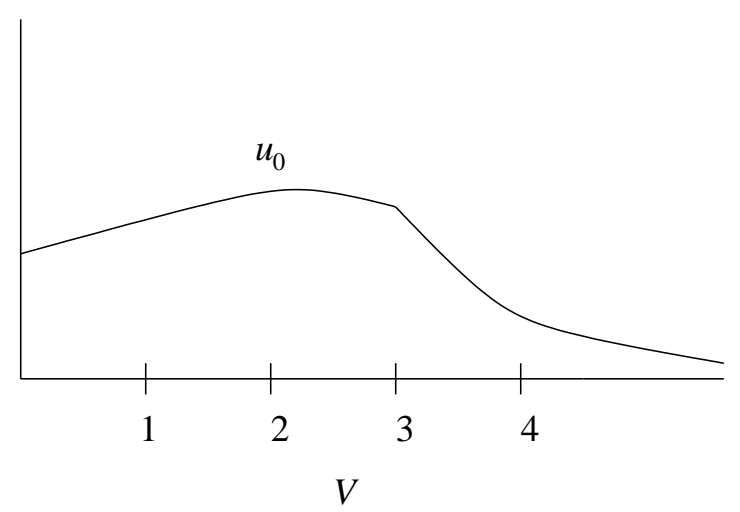

FIG. 11: Schematic plot of the equilibrium dimerization $\lambda u_{0}$ versus interaction at fixed $K_{0}$.

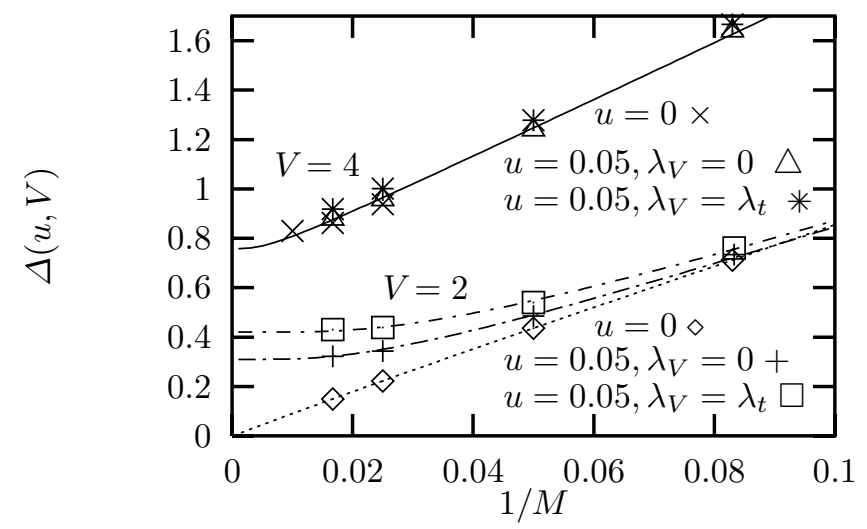

FIG. 12: Gap versus inverse system size for various interaction strengths and dimerizations. The fits are extrapolations, as mentioned in the text, and are meant as "guide to the eye". 